\title{
Lower limb symmetry index (LLSI) pre- and post-reconstruction of the ACL: a controlled study
}

\author{
Luma Soares Lustosa', Nyck Douglas Claro Pereira1, José Jamacy de Almeida Ferreira', Palloma Rodrigues de Andrade',
} Heleodório Honorato dos Santos ${ }^{1,2}$

\begin{abstract}
Background: The anterior cruciate ligament $(A C L)$ lesion causes a deficit in joint stability and mobility, trophism and muscular strength, generating asymmetries between the lower limbs. Objective: To verify the effect of a physiotherapeutic protocol on the Lower Limb Symmetry Index (LLSI) and the correlation between strength and EMGs, pre and post reconstruction of the ACL. Methods: Twenty subjects (10 ACLrg + 10 CONTg) were evaluated regarding isometric force and electrical activity of knee extensors, knee flexors and hip abductors. Results: A significant increase $(\mathrm{P}<0.01)$ in knee extension and flexion strength and hip abduction strength were observed both for the affected limb and non-affected limb. Regarding the LLSI, a significant increase was observed for knee extension and hip abduction movements in the pre- and post-treatment comparison, and between ACLrg X CONTg $(P<0.01)$ for the knee extension movement in the pre-reconstruction phase of the ACL. A very strong correlation $(r=0.945 ; P<0.01)$ was also observed between the LLSI strength X EMGs during knee extension, pre- and post-reconstruction surgery. Conclusions: Six months after reconstruction of the $A C L$, there was an increase in strength and EMG activity of the knee flexor, knee extensor and hip abductor muscles, leveling the LLSI between ACLrg and the CONTg, however, with a significant correlation between the two variables (strength X EMGs) for only one of the three movements (knee extension).
\end{abstract}

Keywords: Knee joint; Torque; Electromyography; Physiotherapy.

\section{INTRODUCTION}

The anterior cruciate ligament ( $A C L)$ is a fundamental structure for the primary restraint of anterior tibial under the femur ${ }^{(1)}$. It is frequently injured while playing sports that require pivoting movements, changes in direction, quick stops and jumps ${ }^{(2,3)}$, leading to lower joint stability and mobility, muscular hypotrophy, reduced strength and deficit in the sensorimotor integrity of the knee ${ }^{(4)}$.

In order to restore the anatomical and functional stability of this joint, treatment is often surgical and it aims to put the individual back at the level of activity prior to surgery, resuming their quality of life $\mathrm{e}^{(5)}$.

Among the aforementioned deficits, post-injury muscle weakness after $A C L$ reconstruction is an important factor to be considered, as it generates imbalances between muscle groups (agonists/antagonists) and between limbs (affected/non-affected), causing persistent asymmetries ${ }^{(6)}$ which can result in below-ideal performance for their return to activities of daily living (ADL) and sports practice, thus increasing the risk of re-injury of this ligament ${ }^{(7-8)}$.
Therefore, the presence of asymmetries in lower limb strength, even after a rehabilitation period, should be considered in subjects who have undergone $\mathrm{ACL}$ reconstruction. Consequently, dynamic assessments that measure the asymmetries and lower limb movement control can help identify possible motor deficits or overloads ${ }^{(9)}$, and the earlier the evaluation of these motor responses during the rehabilitation process, the earlier adjustment procedures can be proposed in order to assist the correct prescription ${ }^{(9)}$, considering that even when subjects perform physical therapy after ACL reconstruction they present asymmetries between lower limbs when compared to a control group ${ }^{(10)}$.

Some studies ${ }^{(11-13)}$ that analyzed the correlations between strength and the amplitude of lower limb surface electromyogram (EMGs) have shown weak correlations between these two variables, indicating a weak correlation between the increase in strength provided by adopting some training programs and the amplitude of the electromyographic signal.

Corresponding author: Heleodório Honorato dos Santos. Avenida monsenhor Odilon Coutinho, 191 - apto. 402. Atlantis Home Service - Cabo Branco. João Pessoa, PB - CEP: 58045-120. E-mail: dorioufpb@gmail.com; Fone: (83) 996137900

1 Universidade Federal da Paraíba/UFPB, João Pessoa, Paraíba, Brasil.

Full list of author information is available at the end of the article.

Financial Support: nothing to declare.

Submission date 28 February 2018; Acceptance date 03 July 2018; Publication date 11 October 2018 
On the other hand, Judge et al. ${ }^{(14)}$ and Andersen et al. ${ }^{(15)}$ found strong and moderate correlations between the strength and the EMG signal of the lower limbs (thigh) after 4 to 6 months of resistance training in athletes and healthy active subjects, respectively, showing that although the literature agrees on the asymmetries, there are conflicting studies regarding the correlation between strength and EMGs of the knee musculature, even after post-surgical ACL rehabilitation.

Thus, the objective of the present study was to verify the effect of a physiotherapeutic protocol in the Lower Limb Symmetry Index (LLSI) and in the correlation between strength and EMGs, pre- and post-reconstruction of the ACL.

\section{METHODS}

\section{Study type and sample characteristics}

This is an experimental, cross-sectional, controlled study performed at the Laboratory for Studying Balance, Dynamometry and Electromyography (Laboratório de Estudo do Equilíbrio, Dinamometria e Eletromiografia - LEEDE) of the Research Center of Human Movement Sciences (NPCMH) of the Federal University of Paraíba (UFPB) involving subjects with reconstructed lesions of $A C L$ ( $A C L r$ group: $A C L r g$ ) and healthy subjects (Control group: CONTg).

Subjects in the ACLrg met the following inclusion criteria: physically active, with unilateral ACL lesion confirmed by clinical tests (Lachman and anterior drawer) and magnetic resonance imaging (MRI), with predictive surgical reconstruction (graft: semitendinous and gracilis (tendons)/ST+Gr), who did not present any other clinical finding of dysfunction or injury to the contralateral lower limb, vestibular or neurological dysfunctions.

Subjects with similar anthropometric characteristics to the ACLrg, without ligament injury, lower limb trauma or diseases which could compromise or make it impossible to perform the tests were selected by convenience for the CONTg.

Forty-six subjects were initially contacted, of whom 22 were allocated to compose the sample (ACLrg: 12 subjects; CONTg: 10 subjects). However, 2 subjects of the ACLrg were excluded during the study due to dropout, and the present study sample consisted of 20 subjects: ACLrg with 10 subjects (28.3 \pm 7.36 years; $79.0 \pm 12 \mathrm{~kg} ; 1.75 \pm 0.10 \mathrm{~m}$; Body Mass Index $/ \mathrm{BMI}=25.7 \pm 2.4 \mathrm{~kg} / \mathrm{m}^{2}$ ); and CONTg with 10 subjects (28.0 \pm 7.2 years; $77.5 \pm 9.7 \mathrm{~kg} ; 1.73 \pm 0.07 \mathrm{~m}$; Body Mass Index $/ \mathrm{BMI}=27.2 \pm 1.6 \mathrm{~kg} / \mathrm{m}^{2}$ ) who completed all assessments (Figure 1).

After the study was approved by the Research Ethics Committee of the Science and Health Center of the Federal University of Paraíba (CEP/CCS/UFPB), under protocol 0121/2014, CAAE: 30168614.8.0000.5188, all the subjects were instructed regarding the procedures and signed a Free and Informed Consent Form (ICF), according to Resolution
$466 / 2012$ of the National Health Council (NHC) and the Declaration of Helsinki.

\section{Procedures}

Anthropometric data were initially recorded (age, height, weight and $\mathrm{BMI}$ ) and the clinical-functional evaluation was carried out (anamnesis, physical examination, inspection, palpation, muscular conditions and mobility, special tests, complementary examinations), followed by an assessment of isometric strength and electromyographic (EMG) activity of knee flexors and extensors, and hip abductors in the pre- and post- reconstruction phase of the ACL.

\section{Dynamometry}

A 5-minute warm-up was performed on a stationary bicycle $(25 \mathrm{~W})$ at $20 \mathrm{~km} / \mathrm{h}$ Prior to the strength test, according to Earl et al. ${ }^{(16)}$ and Linnamo et al. ${ }^{(17)}$.

The maximum isometric strength measurement was collected with the subject sitting in a Bonett chair (adapted) with their trunk (supported on the backrest), pelvis and thigh of the lower limb to be evaluated stabilized by containment belts on the legs, keeping the knee at a $60^{\circ}$ angle for the extension strength, and at a $30^{\circ}$ angle for the flexion strength $^{(13)}$. The subjects were instructed to hold the lateral support of the seat firmly throughout all the test procedures so as not to compensate the movements.

In addition, the maximum isometric strength of the hip abduction movement was recorded with the subject in lateral decubitus position on a stretcher, with the limb to be tested at full extension $\left(0^{\circ}\right)$.

For muscle strength measurements, both the affected limb $(A L)$ and the non-affected limb (NAL) were recorded using a portable digital dynamometer (model DD-300, Instrutherm Ltd., Brazil) considering a series of 3 maximum voluntary isometric contractions (MVIC), each maintained for 5 seconds with an interval of 1 minute between them, in which subjects received verbal stimulus ("strength, strength, strength") from the evaluator, with the mean of the 3 MVICs obtained for each subject being considered for the purpose of calculations ${ }^{(13)}$.

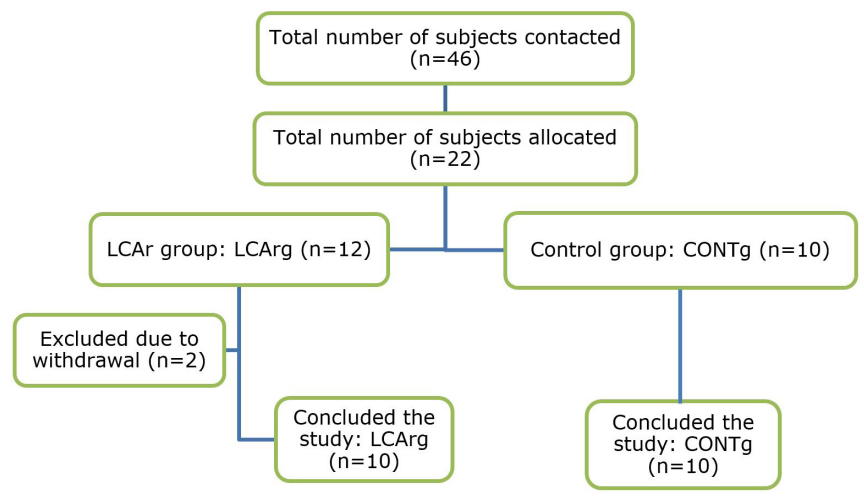

Figure 1 - Flow Diagram of the Sample 


\section{Surface electromyography (EMGs)}

The electrical activity of the vastus medialis (VM), vastus lateralis (VL), semitendinosus (ST), biceps femoris (BF) and gluteus medius (Gmed) muscles were recorded concomitant to the strength assessment using an 8-channel, Bluetooth, electromyograph (model W4X8, Biometrics Ltd., UK) with the following technical characteristics: hardware with 12-bit analog-to-digital (A/D) conversion board; amplifier with gain of 1000 times; bandpass filter from 20 to $500 \mathrm{~Hz}$ (2nd order Butterworth); common mode rejection ratio (RRMC) $>100 \mathrm{~dB}$; signal noise ratio $<3 \mu \mathrm{V}$ RMS; $10^{9} \mathrm{Ohms}$ impedance; surface electrodes, bipolar, active, simple differential, with a 20-fold pre-amplification, fixed according to the Surface Electromyography for the Non-Invasive Assessment of Muscles - SENIAM ${ }^{(19)}$, reference electrode and a DataLOG software for sampling and analysing signals with a sampling frequency of $1000 \mathrm{~Hz}$.

For the VL muscle, the electrode was positioned at $80 \%$ of the line between the anterior superior iliac spine (ASIS) and the joint space at the anterior border of the lateral ligament. For the VM muscle, the electrode was positioned at $80 \%$ of the line between the ASIS line and the joint space in front of the anterior border of the medial ligament. For the BF muscle, it was positioned at $50 \%$ on the line between the ischial tuberosity and the lateral epicondyle of the tibia. For the ST muscle, it was positioned at $50 \%$ on the line between the ischial tuberosity and the medial epicondyle of the tibia, while for the Gmed muscle it was positioned at $50 \%$ over the iliac crest line to the trochanter ${ }^{(19)}$. The reference electrode was fixed to the lateral malleolus of the contralateral limb for all evaluations.

Randomization between the affected $\operatorname{limb}(A L)$ and the non-affected limb (NAL) was carried out (www.randomization. com) prior to data collection for recording both isometric strength and EMG activity.

The following formula, according to Carpes et al. (20), was used for the LLSI analysis with respect to strength and electromyographic signal $(E M G s): L L S I=(N A L-A L): 1 / 2$ $(A L+N A L) \times 100$.

\section{Treatment protocol}

a) $1^{\text {st }}$ week: metabolic exercises, RICE protocol (Rest, Ice, Compression, Elevation), voluntary isometric contraction, patellar mobilization, active mobilization, improvement in the range of motion (ROM) and partial weight support with crutches;

b) $2^{\text {nd }}$ and $3^{\text {rd }}$ weeks: exercise bike (10 minutes), improvement of the ROM ( $\cong 10^{\circ}$ per week), muscle strengthening (calf, thigh and hip), correction of dynamic valgus (unipodal support in front of the mirror) and gait training;

c) $4^{\text {th }}$ and $5^{\text {th }}$ weeks: exercise bike (10 minutes), improvement of the ROM ( $\cong 10^{\circ}$ per week), gradual increase in load for muscle strengthening of the calf, thigh and hip (table extensor and flexor, leg press, mini-squat, etc.), one-legged support and gait training (on the mat: sensory-motor training);

d) $6^{\text {th }}$ and $7^{\text {th }}$ weeks: exercise bike (10 minutes), improvement of the ROM ( $\cong 10^{\circ}$ per week), gradual increase of load for muscle strengthening of the calf, thigh and hip (table extensor and flexor, leg press, mini-squat, chair rising on unipodal support, etc.), step (up and down) and bipodal motor sensory training (plank and trampoline);

e) $8^{\text {th }}$ and $9^{\text {th }}$ weeks: maintenance of previous exercises with gradual increase of load; exercises on the elastic trampoline (bipodal support) and starting straight-line trotting/jogging;

f) $10^{\text {th }}$ and $11^{\text {th }}$ weeks: maintenance of previous exercises with gradual increase in load; strengthening of the posterolateral hip complex, exercises on the elastic trampoline (unipodal support) and starting straight-line and lateral trotting/jogging;

g) $12^{\text {th }}$ to $16^{\text {th }}$ weeks (third and fourth months): maintenance of previous exercises with gradual increase in load, straight-line and lateral jogging and bipodal plyometric training;

h) $17^{\text {th }}$ to $24^{\text {th }}$ weeks (fifth and sixth months): previous exercises, straight, lateral and zigzag trotting/jogging, with acceleration and deceleration, crossovers, spinning (pivoting), unipodal plyometric training, jumps (single and triple) and sports gestures.

It is worth pointing out that the same treatment protocol described above was adopted for all subjects in the ACLr group, which was applied by 2 students in their last year of the Physiotherapy course of the UFPB, under the supervision of 1 professor from the same institution with extensive experience in the post-surgical rehabilitation of ACL.

\section{Statistical analysis}

Data were processed and analyzed using the Statistical Package for the Social Sciences (SPSS - 20.0). The data normality test (Shapiro Wilk) was initially performed, then followed by: the Student's t-test (paired) to compare pre-X post-treatment evaluations; Student's t test (independent) and Wilcoxon test to compare the groups (ACLrg x CONTg); Intraclass correlation test (ICC) to correlate groups (ACLrg $x$ CONTg); and Pearson's test to correlate the variables (Strength $X E M G)$, considering a significance level of $\alpha \leq 0.05$.

To analyze the correlation coefficient, the following classification was considered: null $=0.0$; weak $=0.01$ to 0.3 ; regular $=0.31$ to 0.6 ; strong $=0.61$ to 0.9 ; verystrong $=0.91$ to 0.99 ; and complete $=1.0^{(21)}$.

\section{RESULTS}

Regarding anthropometric data, the intraclass correlation test (ICC) showed very strong correlations in the comparison between ACLrg and CONTg for: age (ICC=0.996; $P<0.001)$, 
weight (ICC=0.970; $\mathrm{P}<0.001)$; $\mathrm{BMI}(\mathrm{ICC}=0.951 ; \mathrm{P}<0.001)$; and a strong correlation for height (ICC $=0.871 ; P<0.003)$, thus confirming that the groups are quite similar and have complete conditions to be compared.

A significant increase $(P<0.01)$ of $31.4 \%$ in knee extension strength and of $18 \%$ in knee flexion strength, in addition to an increase of $23.3 \%$ in the hip abduction strength were observed in comparing the pre $x$ post-ACL reconstruction in the $A L$; while these increases in strength for the NAL were of $26.3 \%, 15.4 \%$ and $14.9 \%$, respectively (Table 1 ).

Regarding the comparison between lower limb strength symmetry index (LLSI) for knee extension and flexion movements and hip abduction, pre- and post-ACL reconstruction, it was observed (Table 2 ) that the knee extension ( $P=0.001)$ and hip abduction ( $P=0.005$ ) were highly significant; however, the difference for the knee flexors, despite lower values, was not significant $(\mathrm{P}>0.05)$.

In comparing the LLSI between ACLrg and CONTg (Table 3), the Student's t-test (independent) showed a highly significant difference $(P<0.01)$ for the knee extension movement in the pre-ACL reconstruction phase.

Regarding the correlations between the LLSI strength and the isometric EMGs signal (strength X EMGs) for the 3 analyzed movements (knee extension and flexion and hip abduction), the Pearson test only showed a very strong correlation between the extension strength pre-surgery and the EMGs signal post-reconstruction of knee extensors ( $r=0.945$; $\mathrm{P}<0.001)$. The correlations were either weak (0.01 to 0.3$)$ or regular (0.31 to 0.6$)$ for all other comparisons, but with no statistical significance $(P>0.05)$.

\section{DISCUSSION}

The results of the present study evidenced the effect of physiotherapeutic treatment by increasing the strength in the knee flexor and extensor muscles and hip abductors at 6 months post-surgical reconstruction of the ACL, presenting a similar LLSI to the CONTg.

A significant gain in knee extension and flexion strength as well as hip abduction was observed in the pre versus post-reconstruction comparison of the $A C L$, both in the $A L$ and in the NAL. This is due to the fact that after the surgical procedure, there is (of course) a tendency for the patient to protect the operated limb by reducing the weight load on $\mathrm{it}^{(6)}$. Moreover, current protocols emphasize performing bilateral, multi-joint (closed-chain) exercises, since in addition to being safer they are also more functional, thus contributing to the strength gains in both the $A L$ and the and $N A L^{(22)}$.

In comparing the LLSI between the groups (ACLrg and CONTg), a significant deficit in knee extension movement was observed in the pre-ACL reconstruction phase, corroborating the affirmation by Lobato(23) that the presence of ACL lesion revealed variable-order deficits in the affected limb when

Table 1 - Average Power Peak (Newton) knee extensor and flexor and hip abductor, pre and 6 months after ACL reconstruction

\begin{tabular}{|c|c|c|c|c|c|c|}
\hline \multirow{2}{*}{ Movements } & \multicolumn{2}{|c|}{$A L$} & \multirow{2}{*}{ P Valor } & \multicolumn{2}{|c|}{ NAL } & \multirow{2}{*}{ P Valor } \\
\hline & Pre & Post & & Pre & Post & \\
\hline Knee Extension & $196.6 \pm 35.8$ & $286.7 \pm 55.3$ & 0.001 & $277.0 \pm 80.1$ & $375.7 \pm 70.9$ & 0.001 \\
\hline Knee Flexion & $267.0 \pm 71.4$ & $325.6 \pm 67.2$ & 0.009 & $296.9 \pm 72.2$ & $343.0 \pm 84.5$ & 0.003 \\
\hline Hip Abduction & $152.6 \pm 18.9$ & $198.8 \pm 36.7$ & 0.003 & $174.7 \pm 39.7$ & $205.3 \pm 47.6$ & 0.005 \\
\hline
\end{tabular}

Note: Legend: $\mathrm{AL}=$ affected limb. $\mathrm{NAL}=$ non-affected limb; Note: Student $\mathrm{t}$ test (paired).

Table 2 - LLSI of extensor and flexor knee and hip abductor force, pre and 6 months after ACL reconstruction.

\begin{tabular}{lcc}
\hline \multicolumn{1}{c}{ Movements } & Pre & Post \\
\hline Knee Extension & $23.7 \pm 4.2$ & $8.9 \pm 7.4$ \\
Knee Flexion & $12.3 \pm 4.0$ & $10.7 \pm 8.0$ \\
Hip Abduction & $12.2 \pm 10.8$ & $9.001 \ddagger$ \\
\hline
\end{tabular}

Note: Legend: LLSI = lower limb symmetry index. Note: $\ddagger=$ Student's $t$ test (paired); $\diamond=$ Wilcoxon test.

Table 3 - LLSI of extensor and flexor knee and hip abductor force (LCArg x CONTg), pre and 6 months after ACL reconstruction

\begin{tabular}{|c|c|c|c|c|c|c|}
\hline \multirow{2}{*}{ Movements } & \multicolumn{2}{|c|}{ Pre } & \multirow{2}{*}{ P Valor } & \multicolumn{2}{|c|}{ Post } & \multirow{2}{*}{ P Valor } \\
\hline & LCArg & CONTg & & LCAg & CONTg & \\
\hline Knee Extension & $23.7 \pm 4.2$ & $7.4 \pm 8.0$ & 0.001 & $8.9 \pm 7.4$ & $7.4 \pm 8.0$ & 0.673 \\
\hline Knee Flexion & $12.3 \pm 4.0$ & $8.8 \pm 7.0$ & 0.191 & $10.7 \pm 8.0$ & $8.8 \pm 7.0$ & 0.586 \\
\hline Hip Abduction & $12.2 \pm 10.8$ & $12.1 \pm 5.6$ & 0.984 & $9.1 \pm 7.2$ & $12.1 \pm 5.6$ & 0.316 \\
\hline
\end{tabular}

Note: Legend: LLSI = lower limb symmetry index. Note: Student's t test (independent). 
compared to a control group (external) due to a phenomenon defined as arthrogenic muscle inhibition, which is a reflex of inhibiting the muscles around the knee when there is damage to the joint, thus reducing the muscular strength of the quadriceps ${ }^{(24)}$.

By comparing the limb strength symmetry index (LLSI) for knee extension and flexion movements and hip abduction pre and post-ACL reconstruction, a highly significant increase was observed for knee extension and hip abduction compared to pre-intervention values. However, although these values were (also) low for the knee flexors, no significant difference was found. This is partially in agreement with Kaminska ${ }^{(25)}$, who reported that the isolated knee-ligament system injury significantly reduces the strength of the flexors and extensors and may decline after surgical reconstruction, and deficits in muscle strength may persist for many years post-injury.

Regarding the correlations between the isometric LLSI strength and the EMG signal (LLSI_strength X EMG) for the 3 analyzed movements (knee extension and flexion and hip abduction), a very strong correlation was found between pre-extension strength and EMG of the knee extensors post-reconstruction ( $r=0.945 ; P<0.001)$. This corroborates the study by Brasileiro et al. ${ }^{(26)}$, who evaluated 8 subjects with $\mathrm{ACL}$ reconstruction after an eccentric training in the $A L$, and found an increase in the EMG signal amplitude for the VL and MV muscles in this limb during isometric strength.

The fact that the correlations between the knee flexor and hip abductor strength and the EMG signal were not significant can be justified based on the study by Bond et al. ${ }^{(27)}$, who states that the level of muscle activation is not strongly correlated to muscle strength during contractions; therefore, it is not possible to conclusively determine the effect that differences in neuromuscular activation had on the muscular forces of the lower limbs.

The large LLSI deficit in the ACLrg compared to the CONTg in the pre-reconstruction phase during the knee extension movement can be justified by the fact that the neuromuscular changes after an injury represent a complex clinical state that can manifest itself with inhibition in the musculature around the compromised joint, especially in the extensor muscles ${ }^{(27,28)}$. Thus, ACL injury is associated with deficit in neuromuscular control, leading to reduced proprioceptive information, to the disturbance of the somatosensory system and to a decrease of muscular activation ${ }^{(29,30)}$.

The present study presents the following limitations: 1) the low number of subjects in the sample, which may have influenced the results due to the difficulty in recruiting subjects with $\mathrm{ACL}$ lesion prior to surgery, since they only seek physiotherapy service after performing ligament reconstruction in the vast majority of cases; and 2 ) the fact that the subjects did not perform the physiotherapeutic treatment with the same professional, although all followed the same treatment protocol.

\section{CONCLUSION}

The results of this study generally showed that an increase in the strength and EMG activity of the knee flexor, extensor and hip abductor muscles were observed at 6 months post-reconstruction of the ACL and that LLSI strength was similar to the control group. On the other hand, this demonstrates that the physiotherapeutic protocol was effective in the recovery of these subjects and that they are able to perform activities of daily living (ADLs) and resume practicing sports within the normality; however, a significant correlation between strength and EMGs did not prevail for all analyzed movements.

\section{AUTHORS' CONTRIBUTIONS}

Lustosa LS: Data collection + Preparation of the manuscript; Pereira NDC: Data collection + Preparation of the manuscript; Ferreira JJA: Revision of the manuscript; Andrade PR: Revision of the manuscript; Santos HH: Statistical treatment + Preparation + Revision of the manuscript.

\section{CONFLICTS OF INTEREST}

Nothing to declare.

\section{AUTHORS DETAILS}

2 Programa Associado de Pós-Graduação em Educação Física (PAPGEF) UPE/UFPB, João Pessoa, Paraíba, Brasil.

\section{REFERENCES}

1. Ataides $G$, Oliveira $M$, Lobo Junior $P$, Araújo $R$, Henrique $P$, Lima $R$ et al. Resultados clínicos e funcionais da cirurgia de reconstrução do LCA contralateral em médio prazo. Universitas: Ciên Saúde, 2013; 11(1):19-28.

2. Boden BP, Dean GS, Feagin Júnior JA, Garrett Júnior WE. Mechanisms of anterior cruciate ligament injury. J Orthop. 2000; 23(6):573-8.

3. Yu B, Kirkendall DT, Garrett WE. Anterior cruciate ligament injuries in female athletes: anatomy, physiology and motor control. Sports Med Arthrosc Rev. 2002; 10(1):58-68.

4. Bassi FAA, Borges APO, Quemelo PRV. Análise eletromiográfica do músculo reto femoral após lesão do ligamento cruzado anterior: um relato de caso. Rev Ter Man. 2010; 8(S1):97-100.

5. Dambros C, Martimbianco ALC, Polachini LO, Lahoz GL, Chamlian TR, Cohen M. Efetividade da crioterapia após reconstrução do Ligamento Cruzado Anterior. Acta Ortop Bras. 2012; 20(5):285-90.

6. Aragão FA, Schäfer GS, Albuquerque CE, Vituri RF, Azevedo FM, Bertolini GRF. Eficiência neuromuscular dos músculos vasto lateral e bíceps femoral em indivíduos com lesão de ligamento cruzado anterior. Rev Bras Ortop. 2015; 50(2):180-5.

7. Hewett T, Myer GD, Ford KR, Heidt RS Jr, Colosimo AJ, McLean SG. Biomechanical measures of neuromuscular control and valgus loading of the knee predict anterior cruciate ligament injury risk in female athletes: a prospective study. Am J Sports Med. 2005; 33(4):492-501.

8. Paterno MV, Schmitt LC, Ford KR, Rauh MJ, Myer GD, Huang B et al. Biomechanical measures during landing and postural stability predict second anterior cruciate ligament injury after anterior cruciate ligament reconstruction and return to sport. Am J Sports Med. 2010; 38(10):196878.

9. Hartigan E, Axe MJ, Snyder-Mackler L. Perturbation training prior to $\mathrm{ACL}$ reconstruction improves gait asymmetries in noncopers. J Orthop Res. 2009; 27(6):724-9.

10. Marchetti PH, Bucchianico EG, Amore T, Nardi PSM, Gali JC, Uchida MC. Desempenho dos membros inferiores após reconstrução do ligamento cruzado anterior. Motriz. 2012; 18(3):441-8. 
11. Rabita G, Pérot C, Lensel-Corbiel G. Differential effect of knee extension isometric training on the different muscles of the quadriceps femoris in humans. Eur J Appl Physiol. 2000; 83:531-8.

12. McCarthy JP, Pozniak MA, Agre JC. Neuromuscular adaptations to concurrent strength and endurance training. Med Sci Sports Exerc. 2002; 34(3):511-9.

13. Santos $H H$, Hanashiro DN, Ávila MA, Camargo PR, Oliveira AB, Salvini TF. Efeito do treino isocinético excêntrico sobre a razão I/Q do torque e EMGs em sujeitos saudáveis. Rev Bras Med Esporte. 2014; 20(3):227-32.

14. Judge LW, Moreau C, Burke JR. Neural adaptations with sport-specific resistance training in highly skilled athletes. J Sports Sci. 2003; 21:419-27.

15. Andersen LL, Andersen JL, Magnusson SP, Aagaard P. Neuromuscular adaptations to detraining following resistance training in previously untrained subjects. Eur J Appl Physyiol. 2005; 93:511-8.

16. Earl JE, Schmitz RJ, Arnold BL. Activation of the VMO and VL during dynamic mini-squat exercises with and without isometric hip adduction. J Electromyogr Kinesiol. 2001; 11(6):381-6.

17. Linnamo V, Strojnik V, Komi PV. EMG power spectrum and features of the superimposed $\mathrm{M}$-wave during voluntary eccentric and concentric actions at different activation levels. Eur J Appl Physiol. 2002; 86(6):534-40.

18. Hermens HJ, Freriks B, Disselhorst-Klug C, Rau G. Development of recommendations for SEMG sensors and sensor placement procedures. J Electromyogr Kinesiol. 2000; 10(5):361-74.

19. Stegeman DF, Hermens HJ. Standards for surface electromyography: the European project "Surface EMG for non-invasive assessment of muscles (SENIAM)" 2007:108-12. Retrieved from: www.biopac.com/knowledgebase/where-do-i-place-electrodes-sensors-transducers/

20. Carpes FP, Mota CB, Faria IE. On the bilateral asymmetry during running and cycling e A review considering leg preference. Phys Ther Sport. 2010; 11(4):136-42.

21. Araújo AGF, Barbosa GM, Freire RA, Andrade PR, Ferreira JJA, Santos HH. Fidedignidade das medidas inter e intratestes com goniômetro universal e artrômetro podálico da amplitude ativa de eversão e inversão do tornozelo. Fisioter Pesq. 2014; 21(4):339-45.

22. Luz BS. Análise da simetria em tarefas motoras em sujeitos submetidos à reconstrução do ligamento cruzado anterior. [Dissertação]. São Paulo (SP). Instituto de Psicologia da Universidade de São Paulo, 2009.

23. Lobato DFM. Avaliação subjetiva da função do joelho da sensibilidade proprioceptiva antes e após a reconstrução do ligamento cruzado anterior [Dissertação]. São Carlos (SP). Universidade Federal de São Carlos (UFSC), 2007.

24. Noronha Neta, MI. Correlação entre torque, equilíbrio e função do joelho após reconstrução do LCA. [Dissertação]. Natal (RN). Universidade Federal do Rio Grande do Norte (UFRN), 2009.

25. Kaminska E, Piontek T, Wiernicka M, Cywinska-Wasilewska G, Lewandowski J, Lochynski D. Differences in isokinetic strength of the knee extensors and flexors in men with isolated and combined cruciate-ligament knee injury. J Sport Rehabil. 2015; 24(3):268-277.

26. Brasileiro JS, Pinto OM, Ávila MA, Salvini TF. Functional and morphological changes in the quadriceps muscle induced by eccentric training after $\mathrm{ACL}$ reconstruction. Rev Bras Fisioter. 2011; 15(4):284-90.

27. Bond CW, Cook SB, Swartz EE, Laroche DP. Asymmetry of lower-extremity force and muscle cctivation during knee extension and functional tasks. Muscle \& Nerve, 2017; 56(3):495-54.

28. Palmieri RM, Ingersoll CD, Hoffman MA, Cordova ML, Porter DA, Edwards JE et al. Arthrogenic muscle response to a simulated ankle joint effusion. Br J Sports Med. 2004; 38(1):26-30.

29. Bonsfills N, Gómez-Barrena E, Raygoza JJ, Núñez A. Loss of neuromuscular control related to motion in the acutely $\mathrm{ACL}$-injured knee: an experimental study. Eur J Appl Physiol. 2008; 104(3):567-77.

30. Courtney C, Rine RM, Kroll P. Central somato sensory changes and altered muscles energies in subjects with anterior cruciate ligament deficiency. Gait Post. 2005; 22(1):69-74. 